\title{
LA CONSTRUCCIÓN DE LAZOS \\ DE TRABAJO ASOCIATIVO: ARTICULACIONES DESDE LA EXTENSIÓN UNIVERSITARIA
}

\section{A CONSTRUÇÃO DE PONTES DE TRABALHO ASSOCIATIVO: JUNTAS DA EXTENSÃO UNIVERSITÁRIA}

\section{BUILDING BRIDGES OF COLLABORATIVE WORK: ARTICULATONS FROM OUTREACH UNIVERSITY PROJECTS}

Lía Mabel Norverto*

Franco Andrés Carcedo**

\section{RESUMEN}

El artículo difunde las actividades desarrolladas en los proyectos de extensión universitaria "Trabajo y producción como herramientas para la autonomía y la inclusión"1 y "Lazos de trabajo, producción e inclusión social"2 $^{\prime 2}$ en la UNLPam junto a organizaciones de trabajo asociativo vinculadas a la economía solidaria (cooperativas de trabajo y microcréditos productivos). Nos propusimos diseñar estrategias que favorezcan la inclusión social de grupos con dificultades de inserción laboral y socio-productiva, promover el intercambio de saberes entre ámbitos académicos y otros espacios de trabajo de la comunidad donde estudiantes, graduados/as y docentes interactuemos en forma comprometida con nuestro entorno. Como la población de estudio e intervención presenta diversas particularidades, proponemos un abordaje interdisciplinario y una metodología participativa, desde la investigación - acción, donde los diagnósticos de necesidades se van realizando en el campo y con los actores involucrados, mediante la construcción conjunta de aprendizajes que fortalezcan la autonomía de los/as trabajadores/as.

Palabras clave: extensión; investigación; interdisciplina; autogestión; trabajo.

\section{RESUMO}

O artigo difunde as atividades desenvolvidas nos projetos de extensão universitária "Trabalho e produção como ferramentas para a capacitação e inclusão" e "Laços de trabalho, da produção e da inclusão social" na UNLPam com organizações que trabalham em parceria ligadas à economia solidária (cooperativas de trabalho e microcrédito produtivo). Propusemos desenhar estratégias que promovam a inclusão

\footnotetext{
* Professora da Universidad Nacional de La Pampa (UNLPam), La Pampa - Argentina. E-mail: Inorverto@gmail.com

** Professor da Universidad Nacional de La Pampa (UNLPam), La Pampa - Argentina. E-mail: francocarcedo77@gmail.com

1 Proyecto de Extensión Universitaria correspondiente a la Secretaría de Cultura y Extensión Universitaria de la UNLPam, período de ejecución 2013-2015. Evaluación externa.

2 Proyecto presentado en la 12a Convocatoria del Año 2012, Secretaría de Políticas Universitarias. Módulo Socio-Productivo, evaluado, seleccionado y financiado por la SPU (Secretaría de Políticas Universitarias). Programa de Promoción de la Universidad Argentina. Ministerio de Cultura y Educación. Resolución Ministerial. 317-12. SPU.
} 
social de grupos com dificuldades em encontrar emprego e sócio-produtivas, promover o intercâmbio de conhecimentos entre as universidades e outros espaços de trabalho da comunidade onde os alunos, graduados/as e professores interagem de maneira comprometida com o nosso ambiente. À medida que a população de estudo e intervenção tem várias particularidades, propomos uma abordagem interdisciplinar e uma abordagem participativa, a partir de pesquisa - ação, em que as avaliações das necessidades estão sendo feitos no campo e com as partes interessadas, por meio da construção conjunta de aprendizagem, fortalecendo a autonomia do/as trabalhadores/as.

Palavras-chave: extensão; pesquisa; interdisciplinar; autogestão; trabalho.

\begin{abstract}
This article shares the experience of the activities of the outreach university projects "Work and production as tools for empowerment and inclusion" and "Ties of work, production and social inclusion" in the UNLPam with organizations working in partnership linked to the economy solidarity (labor cooperatives and productive microcredit). The projects aimed at designing strategies that promote social inclusion of groups with difficulties in finding employment, as well as socio-productive strategies and to promote the exchange of knowledge between academia and other community workplaces where students, graduates and teachers are committed to environmental issues. As the study population and intervention has several particularities, the projects propose an interdisciplinary approach and a participatory approach, from action research in which needs assessments are done in the field and with stakeholders, through joint construction of learning, thus strengthening the autonomy of workers.
\end{abstract}

Keywords: outreach projects; interdisciplinary; self-management; work.

\title{
Presentación
}

Las problemáticas de desocupación, subocupación y precariedad laboral, se consideran uno de los principales problemas que tuvo que afrontar Argentina a fines del siglo XX y principios del XXI. Por ello la temática laboral - y su estrecha vinculación como espacio vital para ejercer la ciudadanía - es la que origina estos proyectos.

Al calor de la crisis, surgieron iniciativas novedosas que despertaron interés e interrogantes en las ciencias sociales como nuevas formas de organización del trabajo, de la economía, de la política y de la sociedad. Experiencias autogestivas, recuperación de empresas, cooperativas de trabajo, microcréditos productivos y otras iniciativas con modalidades asociativas en actividades laborales emergieron - en su gran mayoría - como respuesta a procesos de quiebra de empresas y como coletazos de la profundización de las políticas neoliberales implementadas en Argentina. Miles de trabajadores/as diseñaron formas asociativas para defender o constituir una fuente de trabajo y "aprender" en la marcha a gestionar, administrar, producir, comercializar y decidir sin patrones/as. Al desafío de gestionar y administrar sus emprendimientos, los/as trabajadores/as deben sumar la difícil tarea de consolidar un colectivo humano y generar herramientas de sustentabilidad de sus proyectos laborales, con heterogénea conformación de recursos comunicacionales, económicos, educativos, de género, lo que produce un efecto desigualador y doblemente excluyente del mercado de trabajo.

La dinámica del trabajo y la ocupación en una sociedad es un aspecto ineludible para entender condiciones de vida de una población. El impacto del trabajo en la vida social supera ampliamente la esfera productiva de la misma - desde la cual convencionalmente se lo ha estudiado - e influye en aspectos culturales, motivacionales, emocionales, ambientales, políticos, ideológicos, de la estructura social. Es decir que, participar en el mundo del trabajo, no sólo implica obtener un medio de 
subsistencia, sino construir redes de interacción social y configurar la propia identidad. (Norverto, 2011: 7).

En atención a las múltiples variables que intervienen en las problemáticas sociales - como las que nos ocupan-, nuestra propuesta plantea un abordaje desde miradas interinstitucionales, inter-facultades, inter-disciplinarias e inter-claustros.

Esta tarea nos está resultando enriquecedora en todo sentido, si bien la pensamos originalmente para fortalecer las estrategias de intervención con los/as trabajadores/ as, es una experiencia valiosa de aprendizaje al interior del grupo, ya que no contamos con numerosos espacios donde confluyan distintas miradas, perspectivas, saberes y abordajes. Las producciones y abordajes en el circuito académico suelen "cerrar" sus canales a lo conocido y disciplinar, se observan resistencias y/o dificultades para establecer intercambios entre carreras y disciplinas. El equipo está integrado por miembros de tres facultades (Ciencias Humanas, Agronomía y Económicas y Jurídicas), de las áreas de Geografía, Licenciatura en Negocios Agropecuarios, Historia, Abogacía, CPN, Economía y Sociología. También integra distintos claustros: docentes, estudiantes, graduados/as y una trabajadora administrativa.

Proponemos una metodología participativa, desde la investigación - acción, donde los diagnósticos de necesidades se vayan realizando en el campo y con los actores involucrados. Estos saberes enriquecen nuestros abordajes e interpelan nuestras prácticas académicas, dibujando nuevas maneras de mirar y mirarnos.

La mirada sociológica, de acuerdo con Bauman y May (2007), es una herramienta útil para ensanchar el espectro de nuestra comprensión y visualizar aspectos de la vida humana y social que de otra manera pasarían desapercibidas.

Estas incluyen una pluralidad de experiencias y formas de vida y cómo cada una exhibe y despliega sus formas de conocimiento, demostrando a la vez que no se puede ser una unidad autocontenida y autosuficiente. Muy sencillamente, estamos ligados unos a los otros, aunque en formas diferentes. Este es el desafío, de pensar sociológicamente, porque no detiene sino que facilita el flujo y el intercambio de experiencias (Bauman y May, 2007: 210).

Ciudadanía, derechos, igualdad, democratización, inclusión, autonomía, visibilización, son conceptos que atraviesan toda la propuesta y toman cuerpo trascendiendo las fronteras universitarias. El esfuerzo por visibilizar y desocultar prácticas de desprotección social y segregación, junto a la revalorización de capacidades y autoestima, es una constante que nos enfrentó con nuestras propias "incapacidades" para trabajar con poblaciones por fuera de los circuitos académicos, incitándonos a crear formas innovadoras de intervención y de análisis.

Al investigar poblaciones vulneradas y excluidas del mercado de trabajo formal, nos planteamos interrogantes que delinean los proyectos de extensión, ya que es muy difícil quedarse en el rol de investigadores/as productores/as de conocimiento sin intentar elaborar algunas estrategias de intervención con las mismas.

Estas motivaciones son de índole socio-política-ideológica y surgen al relevar testimonios, o la observación de campo, para producir nuestro aporte al conocimiento disciplinar, lo cual nos coloca en situaciones de incomodidad: ¿Cómo salvar esa distancia y justificar nuestra intromisión en sus mundos de sentido para traducirlos a nuestro mundo académico? Y ¿Cómo evitar la lacerante pregunta de cuáles son los aportes que podemos hacer ante las desigualdades, la pobreza, la marginalidad, la segregación y la exclusión? 
Junto a estas motivaciones ideológicas, también ańadimos las cognitivas, ya que al avanzar en la interrelación con los grupos de trabajadoras/es, advertimos que llegamos a grietas y fisuras que profundizan el conocimiento de las mismas.

Por ello consideramos que la actividad de extensión también tiene que "politizarse" en términos académicos y transformarse mediante su jerarquización, porque mientras trabajar hacia y con el afuera de la universidad, sea concebido como trabajar hacia "los/ as otros/as", o hacer tareas "para la comunidad", contribuiremos a la profundización de las contradicciones entre prácticas y discursos que tomamos como objetos de estudio (Norverto, 2011). Partimos de la convicción de la necesidad de articular docencia, investigación y extensión, para que no queden como pilares o funciones discursivos de la tarea universitaria, sino que nutran y modifiquen formas de hacer y pensar el trabajo universitario, con el propósito de contribuir a la inclusión y cohesión social.

\section{El contexto de estudio e intervención}

La Provincia de La Pampa, ubicada en el centro de la Argentina, contaba en 2010 - último censo nacional realizado - con una población de 318.951 habitantes $(0,80$ del total del país), en una superficie de $143.440 \mathrm{Km}^{2}$. Su densidad poblacional es una de las más bajas del país, es $2,2 \mathrm{~h} / \mathrm{Km}^{2}$. En la Región Noroeste provincial se ubican la mayor cantidad de habitantes, sobre todo en los Departamentos Capital y Maracó, sede de las dos ciudades más importantes y en las mismas se ubican las sedes de la universidad. Las organizaciones con las que trabajamos son de la ciudad capital, cuya población en 2010 fue de 103.241 habitantes (49.722 varones y 53.519 mujeres).

La principal actividad es la agrícola ganadera y, en la ciudad capital, la estructura ocupacional cuenta con una importante proporción de empleo público, ya que es sede de la estructura administrativa del gobierno provincial radica allí. La provincia tiene a su favor menores índices de pobreza, pues que la población con Necesidades Básicas Insatisfechas era en 2010 de 11,7 \% del total, frente a una media nacional de 15,2\%. (Anuario Estadístico, 2015).

En el marco de lo que Tobío (2012) denomina "territorios de la incertidumbre", desde estas propuestas de extensión se busca intervenir en aquellos territorios donde los sujetos que los producen viven en un mundo de incertidumbres vinculadas con las desprotecciones sociales y la falta previsibilidad. Estas situaciones se manifiestan desde los ańos setenta del siglo pasado, cuando el capitalismo comenzó a mostrar problemas en el proceso de acumulación de capital, tendencia recurrente a las crisis y serias dificultades para lograr el crecimiento económico y el desarrollo sostenido. (Harvey, 2007).

En este sentido, la incertidumbre se inscribe en los territorios a partir de la cuestión discursiva, como también en torno a la cuestión material, en tanto lo territorial constituye una dimensión de lo social. Es decir, la incertidumbre posee una existencia concreta, pero también se aloja en las palabras (Tobío, 2012). Desde los proyectos de extensión ${ }^{3}$, se priorizó intervenir en estos territorios, teniendo como experiencia previa los trabajos de extensión y/o de investigación desarrolladas por algunos miembros del grupo en distintas organizaciones de la ciudad de Santa Rosa. Estos colectivos pueden enmarcarse dentro del amplio abanico que incluye la economía social y solidaria. 
Nuestros propósitos fueron diseñar estrategias que favorezcan la inclusión social de grupos con dificultades de inserción laboral y socio-productiva, como también promover el intercambio de saberes y experiencias entre ámbitos académicos y espacios de trabajo de la sociedad civil. Al respecto, son pertinentes las palabras de Horacio Capel cuando reconoce que "no debemos conformarnos con la descripción y el diagnóstico, necesitamos hacer propuestas alternativas" (Capel, 1998: 9). Entre los grupos con los cuales se establecieron vínculos, se encuentran la Cooperativa Telas Propias, Cooperativa Rexiclam, Fundación Trabajo, Emisora Propia Cooperativa de Trabajo y Cooperativa de trabajo Gráfica Visual; ${ }^{4}$ cada uno de ellos con sus dinámicas y dificultades propias.

En esta propuesta participa un equipo de trabajo interclaustro e interdisciplinario, conformado por docentes, graduados/as y estudiantes de las UNLPam interesados/as en reflexionar e intervenir en las problemáticas que enfrentan cada una de las organizaciones mencionadas. Para poder cumplir con los objetivos propuestos, se planteó poner en práctica una metodología participativa, en donde los vínculos e intercambios con las organizaciones se realicen desde una lógica horizontal y enriquecedora para todas las partes. Tin Unwin afirma que la labor de la geografía crítica consiste en poner de manifiesto las desigualdades para convencer a las personas del poder de sus probables repercusiones y para participar activamente en la creación de nuevas formas de organización social y económica. (Unwin, 1995).

Realizamos subgrupos de trabajo - de acuerdo a las investigaciones previas, contactos establecidos e inquietudes propias de la formación/disciplina de cada integrante del equipo - para la coordinación de las intervenciones en cada organización.

Fue necesario un relevamiento en los mismos espacios laborales para identificar las problemáticas específicas de cada organización. Realizamos entrevistas individuales y los aspectos principales fueron sus trayectorias laborales, barrios de residencia, formas alternativas de obtención de ingresos, expectativas laborales, concepción de cooperativismo, forma de ingreso, antigüedad y otros. En todos los casos, se mostró predisposición por parte de los trabajadores/as a responder al cuestionario.

Además de reconocer las dificultades de los grupos, el relevamiento es importante para reconocer los discursos y representaciones de los sujetos sobre sus prácticas laborales y cotidianas. En este sentido, Pierre Bourdieu (2006) plantea que el mundo social es, por una parte, representación y voluntad. Así, la representación del mundo social es:

El producto de innumerables acciones de construcción, siempre ya hechas y siempre por rehacer. Dicha representación se deposita en las palabras comunes, términos performativos que constituyen el sentido del mundo social tanto como lo registran. (Bourdieu, 2006: 187).

Estas representaciones forman parte de los procesos que construyen el espacio social. Constituyen una dimensión específica de los procesos de producción del espacio, al mismo tiempo que representa una instancia del análisis geográfico de tales procesos (Ortega Valcárcel, 2000). Años antes, Lefebvre ya planteaba estas reflexiones cuando reconocía que el espacio como producto social es el resultado "[...] de la acción social, de las prácticas, las relaciones y las experiencias sociales, pero a su vez es parte de ellas. Es soporte pero también es campo de acción. No hay relaciones sociales sin espacio, de igual modo que no hay espacio sin relaciones sociales." (Lefebvre, 1974: 221).

4 Los nombres de las organizaciones son ficticios. 
Las organizaciones que participan del PEU se incluyen dentro de lo que Milton Santos denomina el "circuito inferior" de la economía urbana. En éste la noción de beneficio es diferente respecto a las actividades más modernas del circuito superior. En el primero, "[...] la acumulación de capital no es de interés primordial o no interesa; la tarea primordial es sobrevivir y asegurar la vida familiar cotidiana, así como participar, tanto como sea posible, de ciertas formas de consumo peculiares al moderno estilo de vida." (Santos, 1996: 89).

La conformación de cooperativas de trabajadores/as no siempre se origina en forma voluntaria, en su mayoría se constituyeron ante la necesidad de defensa de la fuente de trabajo y generaron de diversas inquietudes en el grupo humano involucrado, al mismo tiempo que atraviesan muchos obstáculos para salir de la órbita informal de la economía, sin alcanzar ciudadanía económica. En este sentido, acordamos con Tokman (2006) en su planteo sobre la necesidad de saldar una deuda pendiente: la "[...] formalización de los informales", ya que sin ciudadanía económica no hay ejercicio de ciudadanía plena.

En nuestra provincia, y dentro de nuestra ciudad capital, en los relevamientos realizados, observamos ejes comunes que atraviesan los/as trabajadores/as que recurren a la autogestión y otras particularidades propias de la rama productiva o de servicios a la que se dedican, del contexto del período de conformación de la misma, de las trayectorias laborales de sus miembros, las características socio-culturales de los mismos, las tramas vinculares y comunicativas, los conflictos intergeneracionales y las relaciones de género, entre otros.

La consolidación de fuentes laborales autogestionadas por los/as propios/as trabajadores/as significan un valioso aporte a los mismos y sus grupos familiares, así como también al desarrollo de nuevos actores económicos locales, que protagonizan nuevas formas de articular capital y trabajo, construyen modalidades que no se basan en la explotación del trabajo humano.

Estas experiencias también son valiosas porque surgen de abajo y producen sus condiciones mínimas de existencia, la autogestión de sus propias vidas, que han sido desafiadas por el Estado y principalmente por el mercado. Por su carácter de experiencias y de prácticas de lucha sin esquemas, es que requieren una mirada atenta y comprometida con sus procesos y recorridos particulares, que en cada caso, adquiere tonalidades propias.

\section{Los colectivos de trabajo}

Los grupos con los que hemos trabajado, entre los ańos 2013 y 2015, son:

- Cooperativa de trabajo Telas Propias (cooperativa obrera de trabajadoras textiles, ex fábrica de producción de camisas masculinas). Esta se ha conformado en el año 2000 y cuenta con un número que oscila entre las 23- 30 trabajadoras, de ellas una tercera parte son las socias fundadoras de la misma. Es la de mayor antigüedad en la capital pampeana, obtuvieron subsidios para comenzar a funcionar, en un taller alquilado, hasta poder comprarlo. En remate público pudieron acceder a las máquinas, no se les facilitaron las instalaciones ni el equipamiento de la empresa para la que trabajaban.

- Fundación Trabajo. Es una organización no gubernamental que ofrece microcréditos para actividades productivas. La ONG tuvo sus inicios en Argentina en el año 1999 y en La Pampa da sus primeros pasos en el año 2004, en contextos de altos niveles de 
desocupación y con elevados porcentajes de población bajo la línea de pobreza. Establece réplicas en distintos lugares del país y se encarga de facilitar microcréditos sin garantía material a mujeres que no tienen acceso a entidades bancarias ni financieras - ni ellas ni los/as integrantes de su hogar - por carecer de un empleo formal la totalidad del grupo conviviente. Los créditos iniciales con los que comienzan el impulso a sus actividades productivas son de $\$ 1500$, calculado en montos muy bajos posibles de devolver en cuotas semanales por las prestatarias. En la localidad de Santa Rosa la organización concentra su accionar en barrios, cuya población es numerosa y donde se acentúan diversas problemáticas sociales: desocupación, subocupación, bajo nivel educativo, problemas de hacinamiento, trabajo no declarado, entre otros. Los centros han establecido sus sedes en las escuelas de los barrios, quienes prestaron sus instalaciones para las reuniones semanales de los grupos de mujeres. Hay emprendedoras en Zona Norte, en algunos barrios planificados (Santa Rosa) y un centro que funciona con mayor actividad en la localidad de Toay, ubicada a $14 \mathrm{~km}$ de la ciudad capital.

- Emisora Propia Cooperativa de Trabajo Limitada, productores de servicios comunicacionales. Emiten con dos radios: AM y FM. En diciembre de 2008, en un estado de asamblea permanente, los trabajadores decidieron tomar la radio. Unos seis meses después el propietario abandonó la empresa y la asamblea de trabajadores decidió constituirse en un grupo autogestivo, pre-cooperativo, con la finalidad de recuperar la empresa. El 29 de diciembre de 2009 obtuvieron la matrícula como cooperativa de trabajo otorgada por INAES. El 15 de marzo de 2010 empezaron a funcionar formalmente constituidos. Iniciaron la empresa recuperada veintitrés trabajadores y en el período que trabajamos con ellos (2014) eran veintiocho. Los inmuebles donde funcionan las emisoras (estudios en el centro de la ciudad y la planta transmisora) son propiedad del Estado Nacional y mantuvieron los asesores contables y jurídicos que prestaban sus servicios a la firma anterior.

- Cooperativa Rexiclam, tiene sus orígenes en el año 2011. Algunos habitantes del asentamiento El Progreso 5 y un grupo de recuperadores informales autodenominado "trabajadores del relleno", conformaron la cooperativa en la ciudad de Santa Rosa. El primer objetivo fue la incorporación de los clasificadores a la planta de reciclado que el municipio planeaba construir. En su momento fundacional, la cooperativa estaba integrada por 64 personas. En el año 2014 los trabajadores que participaban en la recuperación de residuos eran aproximadamente 30 , dividiéndose en dos turnos diarios.

En el último trimestre del 2013, mediante distintas intervenciones municipales, incorporaron a la planta de separación de residuos a los trabajadores "independientes" y los obligaron a establecer acuerdos que diluyen los intentos de consolidar la cooperativa. Actualmente, la mayoría de los socios originarios se ha retirado de la actividad y se buscaron otras alternativas de generación de ingresos, dada la precariedad en la que se desarrolla la misma. Asimismo, a fines del ańo anterior, los recicladores que se nuclean en la Cooperativa, los "independientes" y el Municipio, firmaron un Acta Acuerdo (Resolución 1463/13), mediante el cual se deja sin efecto el Convenio suscripto el 22 de marzo de

5 El asentamiento está ubicado al Noroeste de la ciudad de Santa Rosa sobre los terrenos fiscales aledaños al relleno sanitario municipal. La ocupación de las tierras se realizó en el año 2007 cuando un hornero primero y un grupo de familias después, se instalan en la zona. Las viviendas del barrio están construidas con chapas, nylon y, salvo algunas excepciones, con ladrillos. El lugar no cuenta con servicios urbanos básicos como electricidad, agua o gas natural, como tampoco centros comerciales o dependencias del Estado. Las intervenciones del municipio se limitan al abastecimiento de agua mediante camiones cisterna, los cuales se acercan al asentamiento solo dos veces por semana. 
2012. La firma de este último Convenio se logró luego de que los principales líderes de la cooperativa ocuparan la sede municipal como forma de protesta ante la negativa del Ejecutivo. De esta forma, con la suscripción del Acta Acuerdo del 20 de diciembre de 2013, el municipio retoma el control del relleno sanitario y de los/as trabajadores que allí se desempeñan.

- Cooperativa de Trabajo Visual: está compuesta por 7 asociados, desde el año 2007. Surgió a partir de los procesos de flexibilización laboral como parte de las estrategias empresariales de reducción de costos laborales. La mayoría de sus asociados eran empleados de una empresa privada dedicada al diseńo e impresión, a los que se les propuso pasar a ser trabajadores independientes monotributistas, perdiendo su condición asalariada. Sin llegar a un acuerdo con el empleador, decidieron desvincularse y asociarse en forma independiente, conformando una cooperativa de trabajo gráfica.

\section{Método y actividades}

En sintonía con Partenio (2010), más allá de las condiciones formales que impone la figura cooperativista, nos interesa analizar la forma que asume la gestión colectiva y la participación de trabajadores/as, los cuales presentan una serie de desafíos, como las funciones de dirección/conducción, tareas de planificación, asignación de responsabilidades, distribución de excedentes, construcción de vínculos con otros actores sociales, regulación de conflictos, selección e incorporación de nuevos asociados/as a las cooperativas.

Los relevamientos que realizamos nos dan cuenta de estas acciones, que van tomando matices y colores particulares en cada organización. Atravesados por cortes generacionales, antigüedad en la misma, trayectorias laborales, niveles educativos, espacios y tiempos de trabajo, tipos de tareas, los cuales adquieren diversas representaciones en la construcción del "espíritu cooperativo" que la mayoría parece anhelar.

Entre las acciones implementadas podemos mencionar:

- relevamientos a fin de conocer las características y necesidades de las distintas organizaciones;

- talleres y encuentros sobre demandas de cada grupo;

- intervenciones en colegios secundarios de nivel medio;

- seguimiento y acompańamiento de los emprendimientos productivos de mujeres;

- diseño de folletería para difundir productos y servicios de las mujeres emprendedoras;

- diseño de una planilla de control administrativo de créditos y cobranzas para la ONG;

- organización de una colecta solidaria en la UNLPam ante emergencia de salud de la hija de un trabajador;

- organización y participación del equipo en encuentros de cooperativas de trabajo; 
- articulación con federaciones que nuclean a cooperativas y trabajadores autogestivos ${ }^{6}$;

- impulso y apoyo en la organización de una federación provincial específica para cooperativas de trabajo que les de visibilidad social y fortaleza política;

- continuidad de trabajo a través de la federación, que se conformó a inicios del 2015. Participación de reuniones mensuales de la misma.

\section{La vinculación inter-institucional y el aprendizaje mutuo}

Con respecto a los vínculos que se generaron con las organizaciones, los grados de integración fueron diversos debido a las diferencias y los tiempos de cada colectivo de trabajo. En algunos casos, la existencia de cuerpos dirigenciales que concentran y arbitran la toma de decisiones, dificultó entablar una comunicación estable con el resto de los/as trabajadores. En este sentido, la posibilidad de participar en los procesos organizativos/ productivos de las cooperativas fue limitado por los miembros más influyentes del grupo. Esta situación fue muy notoria en la cooperativa textil y se pudo intervenir con mayor interacción a partir de cambios en las conducciones de los Consejos de Administración.

En el caso de Rexiclam, los cambios en el Consejo de Administración y la expulsión de asociados comprometidos con la cooperativa resultó desfavorable para iniciar las acciones planificadas. No obstante, se entablaron vínculos con algunos trabajadores interesados en la propuesta de extensión universitaria, los cuales han participado en charlas e intervenciones en colegios secundarios. Quizás la situación de esta cooperativa sea la más compleja a la hora de analizar la organización como tal, debido a que la mayoría de los asociados originarios se alejaron de la organización y los actuales trabajadores no se sienten parte de una cooperativa de trabajo. Asimismo, al ser el municipio quien decide la incorporación de un trabajador a la planta de reciclado, el poder de decisión de los recicladores es acotado.

Una cuestión que se pudo observar en ambas organizaciones es la reticencia a disponer de un tiempo para realizar actividades "no productivas" (como asambleas o talleres). Ello se vincula, en parte, con las características de los liderazgos, la falta de interés del resto del grupo y la percepción de la cuestión organizacional como secundaria en relación a la comercial/productiva. Además, como la realización de asambleas se realiza en pos de mantener la formalidad, no está incorporado en los/as asociados/as la toma de decisiones de manera colectiva. Colaborar en la revisión de esas prácticas fue parte de nuestra tarea y aporte.

Las palabras del presidente de Textiles Pigüé, fábrica textil recuperada y autogestionada por sus trabajadores/as, "Nos consume la cabeza la diaria, la urgente, lo que hay que atender" refleja la situación de las preocupaciones de muchos trabajadores/ as autogestionados/as, en las cuales relegan - por su carácter de postergables, posibles de realizar en otros momentos - los espacios de reflexión, planificación y diálogo conjunto que podría potenciar sus esfuerzos.

6 Fecootra: Federación de Cooperativas de Trabajo FACTA: Federación Argentina de Cooperativas de trabajadores autogestionados

7 Exposición en el " $1{ }^{\circ}$ Encuentro Regional Sudamericano La economía de las/os trabajadores/as", 3 y 4 de octubre de 2014, Cooperativa Textiles Pigüé, Pigüé, Pcia de Buenos Aires. 
Así es como nos ha sucedido llegar a las organizaciones con talleres de trabajo acordados, planificados con antelación y encontramos a las/os trabajadores/as ocupados en sus tareas "productivas", sin poder desarrollar las actividades programadas. Estas situaciones nos enseñan a articular en forma más realista con las formas de autogestión, a respetar tiempos y prioridades de las mismas, y a romper con las visiones "modelizantes" o idealizadas de dichos procesos. Nos sitúa en el escenario concreto y real de sus acciones y prácticas, así como nos muestra que nuestra participación tiene límites.

Esto también podemos vincularlo a las dificultades que enfrentan los/as trabajadores/ as para producir hábitos y prácticas nuevas, los cuales han sido socializados bajo los dictámenes salariales. Las palabras de una trabajadora textil sintetizan con mucha claridad la tensión entre dejar de ser empleadas en relación de dependencia y asumir la gestión de la propia organización: "es difícil sacarse el delantal, es más fácil que alguien te diga qué tenés que hacer" (cooperativista textil). Asumen una gestión no buscada ni deseada, rodeada de incertidumbres, especialmente en los casos de recuperación de empresas. Esto permite ver la relación dialéctica entre necesidades y oportunidades, lo que sitúa estas realidades no previsibles en el plano de la invención, como una acción creativa de los/as trabajadores/as que permite superar las brechas entre el trabajo prescripto - y los hábitos adquiridos en el mismo - y los imprevistos del trabajo real. (Dicapua, Tavella y Valentino; 2013).

Desde las universidades, también estamos en períodos de invención: necesitamos generar espacios de articulación inter-institucional que fortalezcan nuestros saberes y las prácticas de formación para vivenciar la preparación profesional - no sólo del claustro estudiantil - en interrelación con las problemáticas de la comunidad, al mismo tiempo que aprender a "negociar" con intereses en disputa y conflicto. No confundir esos espacios como un escenario libre y desinteresado, sino comprender sus propias relaciones de fuerza, poderes, intereses y conflictos. Esas relaciones de fuerza, muchas veces, entran en colisión con las del trabajo académico y es necesario el aprendizaje mutuo al mismo tiempo que evidenciar los diversos posicionamientos. La desconfianza, el extrańamiento y la invasión en sus propios terrenos, suelen ser obstáculos a tener en cuenta para transitar los conflictos.

Algunos testimonios son elocuentes al respecto: "si no nos van a cobrar, si la propuesta es gratis, está bien..." "vienen muchos y nos hacen relevamientos, ven, observan, nos dicen que nos van a ayudar, pero no pasa nada..." "la verdad es que no entiendo mucho qué quieren hacer, problemas tenemos muchos y dificultades para organizarnos también, pero algunos del grupo son muy cerrados, no sé si van a querer venir fuera de horario..." "no les respondimos antes porque queremos saber bien cuál es su propuesta, qué nos ofrecen" "nos hace falta ayuda, para organizarnos fundamentalmente, es difícil ponerse de acuerdo..."

"no vamos más a capacitaciones o encuentros de cooperativas porque nos llaman y muchas veces es una pérdida de tiempo, se tratan temas que no nos sirven, de otras cooperativas, que no tienen que ver con nosotras..." (trabajadores/as de distintas cooperativas).

El intercambio con las organizaciones de la economía social y solidaria nos coloca en ese interjuego de intereses desiguales, heterogéneos, de relaciones de poder asimétricas y diseminadas por todo el tejido social, que nos ha ayudado a delinear unas primeras ideas:

- Las organizaciones construyen sus propios límites y la posibilidad de intervención está limitada por estos; 
- Esta tarea es un desafío junto con las organizaciones para romper con las prácticas verticales y asimétricas que encierran las "capacitaciones" para la inserción en el mundo de trabajo;

- También el desafío es articular con federaciones, organizaciones políticas y organismos gubernamentales vinculados al sector de la economía social que "representan" al cuerpo de trabajadores, quienes también entran en la escena y se producen conflictos de intereses, de legitimidad y de representatividad;

- Nuestros propios intereses y modos de "transferir" encuentran limitaciones y nos obligan a crear formas constructivas que desanden las convencionales maneras de enseñanza-aprendizaje e investigación;

- Esto posibilita encontrar el carácter político a las experiencias y al saber de lo cotidiano, como una palanca que puede cambiar las formas de organización del trabajo, de la economía y de la producción de conocimiento.

Pierre Bourdieu (2002) incita al rol comprometido de los investigadores con la escucha, creación y búsqueda de resistencia a las políticas neoliberales, desde una apertura del saber colectivamente adquirido y controlado. Si el saber se construye en forma colectiva, no puede ser reducido exclusivamente a las críticas de colegas.

$\mathrm{El}$ investigador no es ni un profeta ni un guía de pensamiento. Debe inventar un rol nuevo que es muy difícil (...) Los investigadores pueden también volcarse a algo nuevo, más difícil: estimular la aparición de condiciones organizacionales de producción colectiva que conduzcan a la creación de un proyecto político ¿Y cuál puede ser el rol de los investigadores en todo esto? Trabajar en la invención colectiva de estructuras que den origen a un nuevo mundo social, es decir, a nuevos contenidos, nuevas metas y nuevos medios internacionales de acción. (Bourdieu, 2002: 156).

Esta tarea es posible mediante una construcción también colectiva, que desafíe nuestras jerarquías y prioridades académicas. Por ello consideramos que la actividad de extensión tiene que "politizarse" en términos académicos y adquirir un papel relevante y valorizado. Estamos convencidos de la necesidad de articular docencia, investigación y extensión, para que no queden como pilares o funciones discursivas de la tarea universitaria, sino que nutran y modifiquen formas de hacer y pensar el trabajo universitario.

Un poco de introspección y de revisión de nuestras propias prácticas nos sería de mucha utilidad en la construcción del camino hacia la integración de la extensión, docencia e investigación que ayude a la inclusión y cohesión social.

\section{Bibliografía consultada}

ANUARIO ESTADÍSTICO. Dirección Provincial de Estadística y Censos. Gobierno de La Pampa, 2015. Disponible en http://www.estadistica.lapampa.gov.ar/images/Archivos/ Anuario2015/Anuario_Estadistico_2015_bajo.pdf

BAUMAN, Z. y MAY, T. Pensando sociológicamente. Buenos Aires: Nueva Visión, 2007.

BOURDIEU, P. Los investigadores y el movimiento social. En Pensamiento y Acción, Editorial Zorzal, Buenos Aires, 2002. 
BOURDIEU, P. Campo de poder y reproducción social. Elementos para un análisis de la dinámica de las clases. Ferreyra, Buenos Aires, 2006.

CAPEL, H. Una geografía para el siglo XXI. En Scripta Nova, № 19, Universidad de Barcelona, 1998.

DICAPUA M.;TAVELLA M.;VALENTINO N. Empresas recuperadas y políticas sociales: presionar, resignificar, inventar, producir. En Detrás de la mirilla. Trabajo y Género en las nuevas formas de asociatividad. Dicapua M.; Mascheroni J. y Perbellini M. (comps). Ediciones del Revés, Rosario, 2013.

HARVEY, D. Espacios del capital. Hacia una geografía crítica. Akal, Madrid, 2007.

LEFEBVRE, H. La producción de l’espace. París: Anthropos, 1974.

NORVERTO, L. De mujeres y saberes: experiencias compartidas entre la investigación y extensión. XI Congreso Iberoamericano de Extensión Universitaria “Integración extensión, docencia e investigación para la inclusión y cohesión social". UNL 22 al 25 de noviembre de 2011.Edición en CD con trabajos completos y Libro de Resúmenes. E-book. ISBN: 978-987-657-706-9, 2011.

ORTEGA VALCÁRCEL, J. Los horizontes de la geografía. Teoría de la geografía. Ariel, Barcelona, 2000.

PARTENIO, F. Las manos, los saberes, las máquinas. Un análisis sobre la organización del trabajo en los elencos productivos de fábricas recuperadas Capítulo 4 en CROSS, Cecilia y BERGER, Matías (comp) La producción del trabajo asociativo. Condiciones, experiencias y prácticas en la economía social, CEIL PIETTE, CONICET, CABA, Ediciones Ciccus, 2010.

SANTOS, M. De la totalidad al lugar. Oikos Tau, Barcelona, 1996.

TOBÍO, O. Territorios de la incertidumbre. Apuntes para una Geografía Social. UNSAM EDITA, San Martín, 2012.

TOKMAN, V. Inserción laboral, mercados de trabajo y protección social. Publicación de las Naciones Unidas, CEPAL, Santiago de Chile, Colección Documentos de Proyecto, 2006.

UNWIN, T. El lugar de la geografía. Cátedra, Madrid, 1995. 